\title{
SÍNTESE DE PARTÍCULAS DE COPOLÍMERO DE ESTIRENO- DIVINILBENZENO MAGNETIZADAS E SUA APLICAÇÃO NA IMOBILIZAÇÃO DE LIPASES
}

\section{R. L. TAVARES FILHO ${ }^{1}$, H. B. S. BENTOํㅡㄴ L. FREITAS, H. F. de CASTRO e P. C. de OLIVEIRA $^{1}$}

${ }^{1}$ Universidade Estadual de São Paulo, Escola de Engenharia Química de Lorena, Departamento de Engenharia Química

E-mail para contato: charles@alunos.eel.usp.br

\begin{abstract}
RESUMO - O objetivo desse trabalho foi sintetizar partículas magnetizadas de poli(estireno-co-divinilbenzeno) (STY-DVB) e avaliar seu potencial como suporte na imobilização da lipase de Candida rugosa via adsorção física. O desempenho do biocatalisador magnetizado preparado foi comparado com o da lipase imobilizada no suporte de STY-DVB não magnetizado. Os suportes foram imobilizados em meio heptano com a proporção de $0,25 \mathrm{~g}$ de lipase para cada $1 \mathrm{~g}$ de suporte. A influência do tamanho das partículas dos suportes foi analisada em relação à atividade hidrolítica e rendimento de imobilização. As características morfológicas dos materiais e de seus respectivos derivados imobilizados foram analisadas por microscopia eletrônica de varredura (MEV). Apesar de ter influenciado nas características morfológicas do suporte, a adição de partículas magnéticas ao copolímero não prejudicou seu desempenho na imobilização, apresentando valores de atividade hidrolítica próximos. O biocatalisador magnetizado apresentou ainda maior rendimento médio de imobilização $(84,8 \%)$ quando comparado ao derivado não magnetizado (64,4\%), indicando grande potencial de aplicação do suporte estudado na imobilização de lipases.
\end{abstract}

\section{INTRODUÇÃO}

Os polímeros magnéticos caracterizam-se como materiais híbridos por apresentarem propriedades de ambos os seus constituintes, uma substância orgânica e uma substância inorgânica. Por apresentarem características de mais de um único constituinte, estes compósitos despertam grande interesse de produção e aplicação (Lee et al., 2003).

Na perspectiva de diminuição dos impactos ambientais causados pelos processos da indústria atual, os polímeros magnéticos aparecem como alternativa para a catálise, tendo em vista a utilização desses materiais como catalisadores de troca iônica ou como suporte para imobilização de enzimas. Resinas à base de estireno e divinilbenzeno estão sendo estudadas devido a algumas propriedades de processo, como a simplicidade de síntese, e do produto final, tais como facilidade de separação, estrutura porosa, resistência mecânica entre outras (Coutinho et al., 2004).

A aplicação de enzimas como catalisadores apresenta diversas vantagens ao processo, como o fato de atuarem em condições brandas de temperatura, $\mathrm{pH}$ e pressão. As lipases 
(triacilglicerol hidrolases, E. C. 3.1.1.3) se destacam dentre estas enzimas devido a sua versatilidade em catalisar diversas reações como hidrólise, esterificação e transesterificação, e apresentam aplicação industrial em diversas áreas, como na indústria farmacêutica e de alimentos. A técnica de imobilização de enzimas em suporte sólido agrega vantagens ao biocatalisador, oferecendo maior resistência e aumento de estabilidade, além de possibilitar sua reutilização (Adlercreutz, 2013).

\section{MATERIAIS E MÉTODOS}

\subsection{Materiais}

Foi utilizada a lipase de Candida Rugosa (tipo VII, Sigma-Aldrich) imobilizada via adsorção física em copolímero de STY-DVB magnetizado e não-magnetizado. Outros materiais utilizados foram n-heptano (Cromoline), polietilenoglicol MM 1500 (Synth), azeite de oliva comercial com baixo teor ácido (Carbonell) e goma arábica em pó pura (Synth).

\subsection{Métodos}

Síntese do copolímero de STY-DVB magnetizado: O suporte de STY-DVB foi sintetizado pela técnica de polimerização em suspensão a $70{ }^{\circ} \mathrm{C}$ por $24 \mathrm{~h}$, utilizando a formulação encontrada na Tabela 1, o agente de suspensão foi constituído de solução aquosa $1 \%(\mathrm{~m} / \mathrm{m})$ de álcool polivinílico (PVA, MM 78000, 88\% hidrolisado, Polysciences Inc.). As condições de síntese do STY-DVB magnetizado permaneceram as mesmas, com adição de $10 \%(\mathrm{~m} / \mathrm{m})$ de magnetita obtida pela co-precipitação dos íons $\mathrm{Fe}^{2+}$ e $\mathrm{Fe}^{3+}$ em meio básico, e modificada com ácido oleico de acordo com a metodologia descrita por Lee et al. (2003). Após a polimerização, as partículas obtidas foram classificadas por peneiramento utilizando peneiras de 24, 35, 42 e 80 mesh (Tyler).

Tabela 1 - Formulação utilizada nas reações de Polimerização

\begin{tabular}{cc}
\hline Reagente & Massa (g) \\
\hline Estireno (Cromoline) & 7,81 \\
Divinilbenzeno (Cromoline) & 7,81 \\
AIBN (MIG química) & 0,2768 \\
Heptano (Cromoline) & 17,18 \\
Toluol (Cromoline) & 17,18 \\
Agente de suspensão & 208,19 \\
\hline
\end{tabular}

Imobilização da lipase microbiana de Candida rugosa nas partículas de STY-DVB e STY-DVB magnetizado: A lipase de Candida rugosa foi imobilizada via adsorção física nos suportes de STY-DVB magnetizado e não magnetizado através do contato da enzima com o suporte $\left(0,25 \mathrm{~g} / \mathrm{g}\right.$ suporte) junto à solução aquosa de $5 \mathrm{~g} \mathrm{~L}^{-1}$ de polietilenoglicol $(100 \mu \mathrm{L} / \mathrm{g}$ suporte), por um período de $24 \mathrm{~h}$ a $4^{\circ} \mathrm{C}$. Após a imobilização, o derivado foi recuperado por filtração a vácuo seguida de lavagem com heptano até umidade inferior a $15 \%$. 


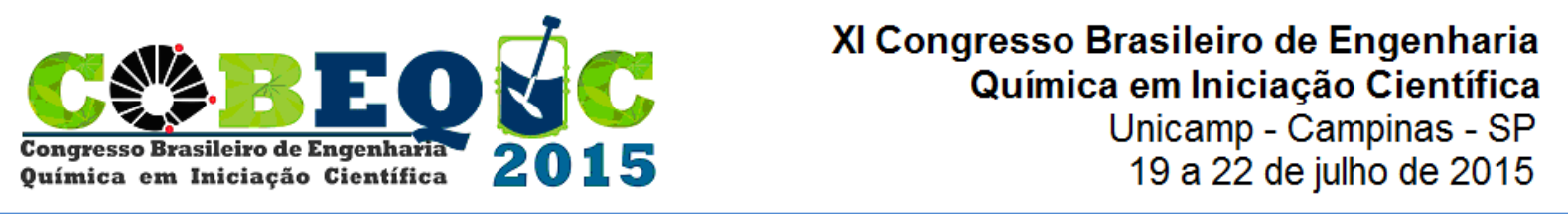

As atividades hidrolíticas da lipase livre e dos derivados imobilizados foram quantificadas pelo método de hidrólise do azeite de oliva (Soares et al., 1999), e o rendimento de imobilização $(\eta \%)$ calculado pela relação de unidades de atividade oferecida para imobilização e a quantidade recuperada pelo derivado imobilizado.

\section{RESULTADOS E DISCUSSÃO}

\subsection{Síntese do Copolímero de STY-DVB Magnetizado}

O polímero magnetizado foi sintetizado com sucesso, apresentando atração por um campo magnético externo (imã), como mostrado na Figura 1. A reação de polimerização ocorreu da mesma forma para os dois suportes, porém, devido à adição de partículas sólidas ao meio reacional (magnetita) na síntese do suporte magnetizado, a velocidade de agitação precisou ser maior para garantir a uniformidade do meio. A modificação da superfície da magnetita com ácido oleico permitiu maior incorporação ao polímero devido a sua maior facilidade de interação com a fase orgânica da polimerização, como explicado por Liu et al. (2006), porém esta modificação da superfície alterou a forma final do polímero, deixando-o amorfo.

Figura 1 - Copolímero magnetizado sofrendo aplicação de um campo magnético externo.

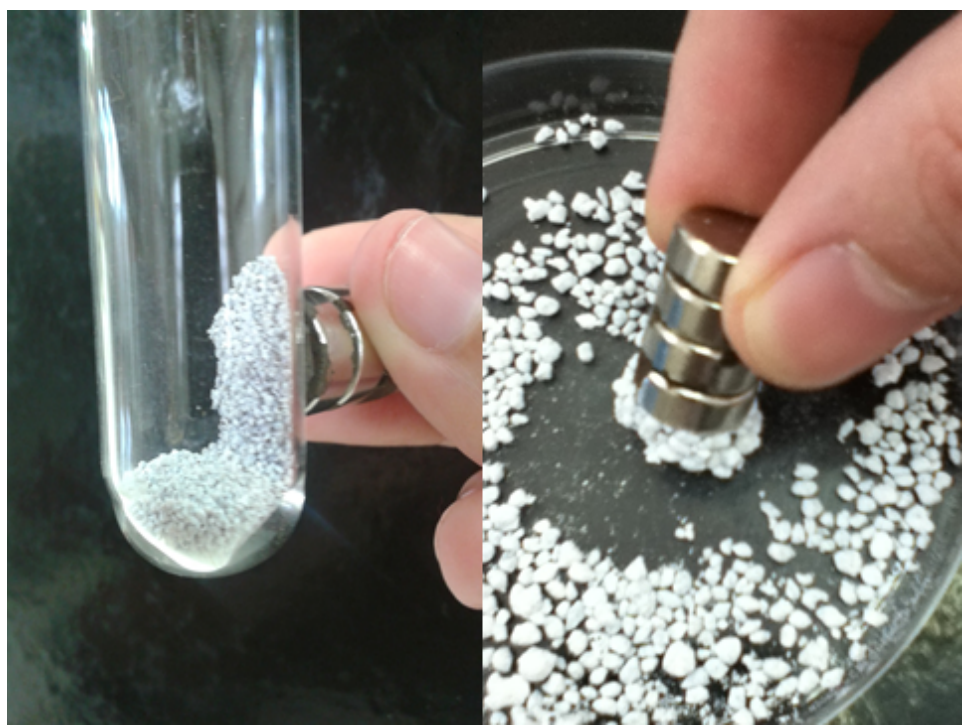

\subsection{Análises de Microscopia Eletrônica de Varredura (MEV)}

As características morfológicas de ambos os suportes e seus respectivos derivados imobilizados foram analisadas por microscopia eletrônica de varredura. Foi possível observar pelas Figuras 2 e 3 que o suporte de STY-DVB apresentou forma predominantemente esférica, ao contrário do suporte magnetizado que se mostrou amorfo. A análise permitiu constatar também que a imobilização da lipase de Candida rugosa nos suportes apresentou as características esperadas, sendo possível visualizar que a enzima encontrou-se aderida à superfície do material. A diferença em relação à uniformidade não influenciou na imobilização. 
Figura 2 - Imagens de MEV: suporte STY-DVB ampliado 100x (a) e ampliado 500x (c) e do derivado imobilizado ampliado 100x (b) e ampliado 500x (d).
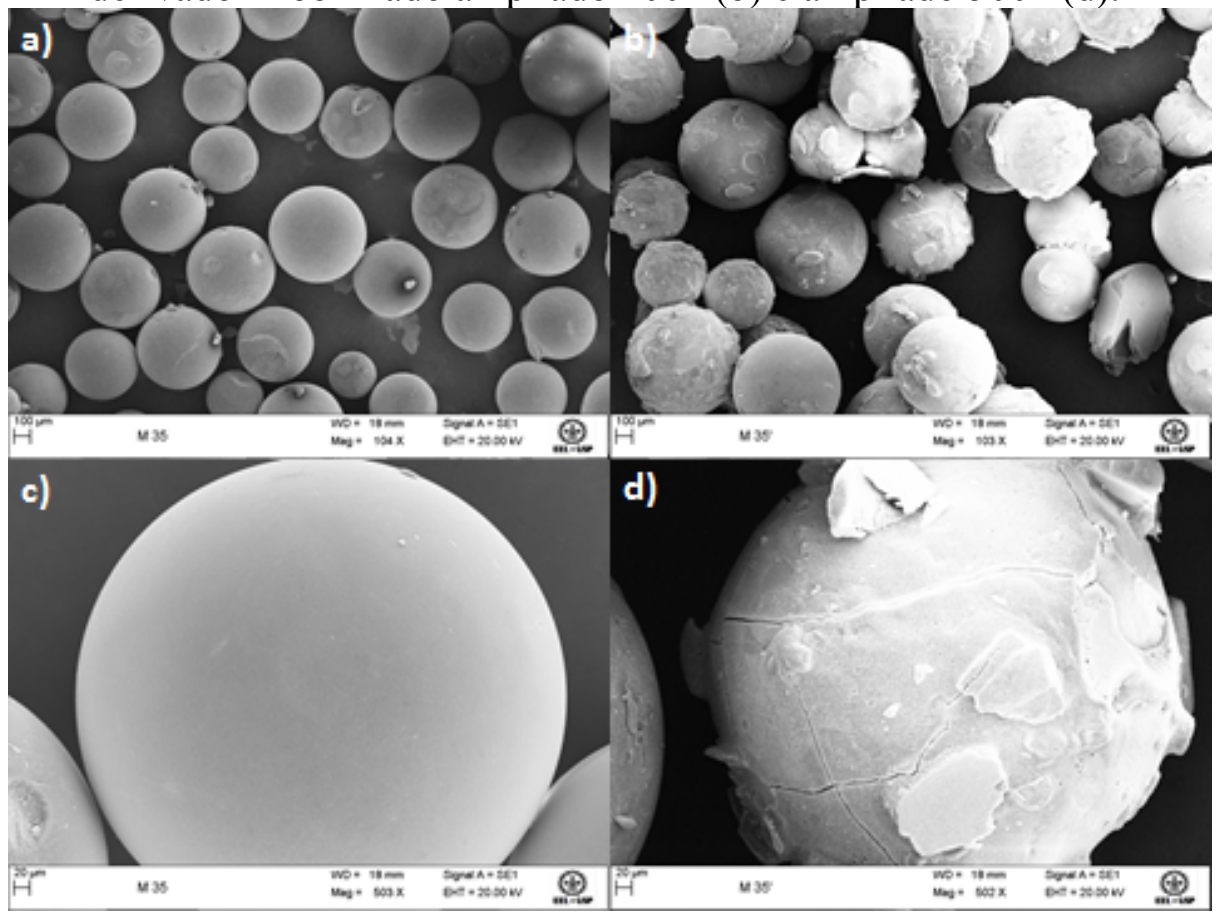

Figura 3- Imagens de MEV: suporte STY-DVB magnetizado ampliado 100x (a) e ampliado 500x (c) e do derivado imobilizado ampliado 100x (b) e ampliado 500x (d).

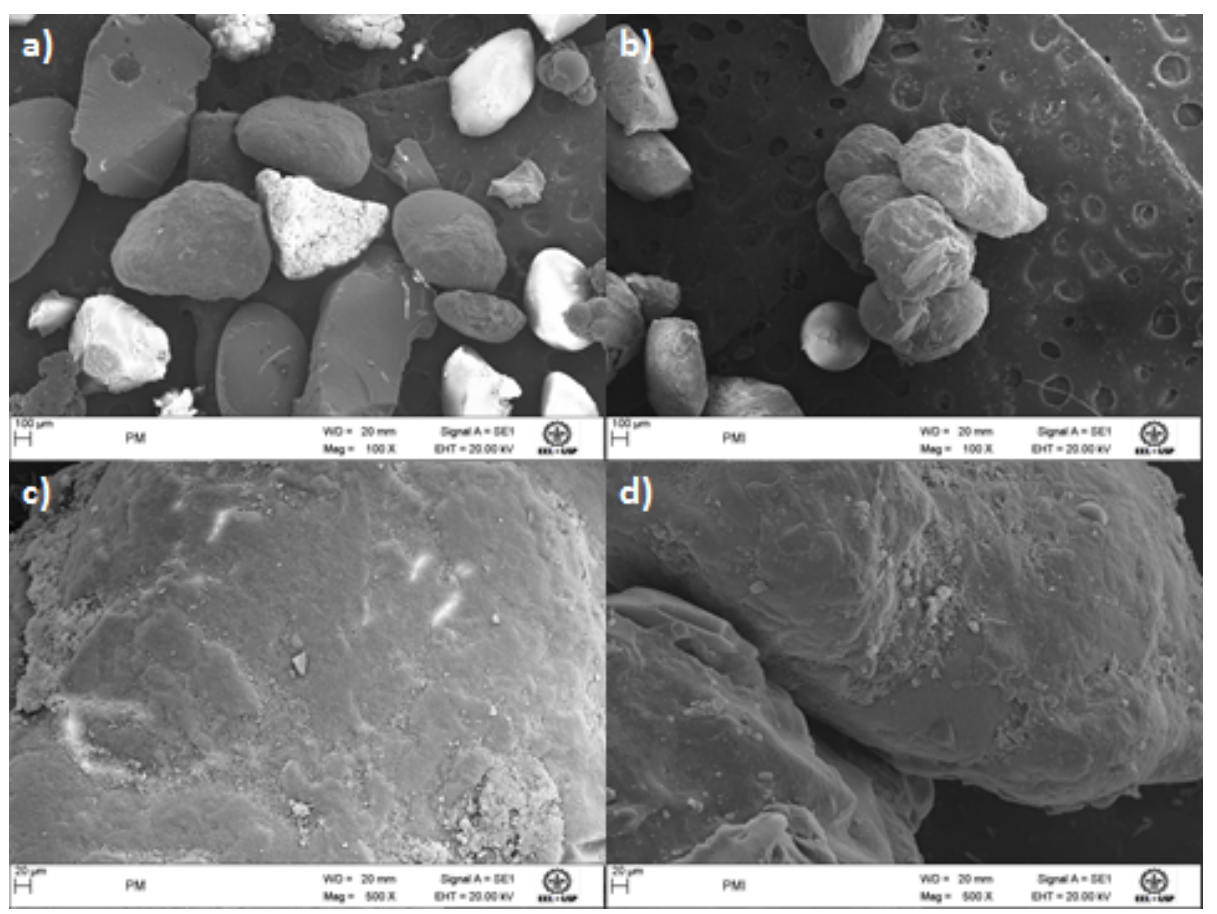




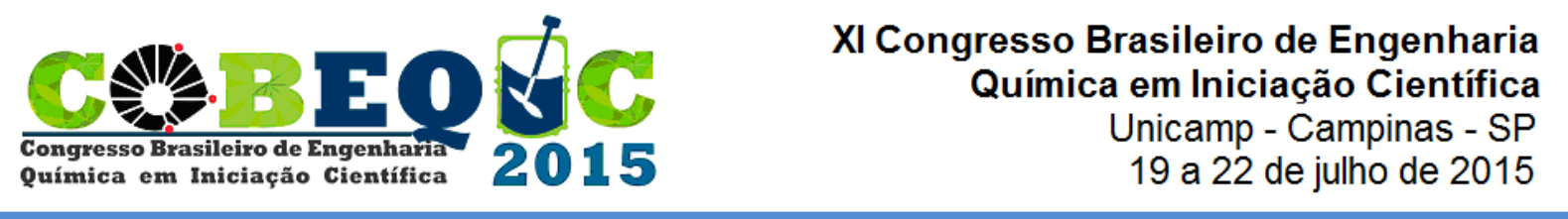

\subsection{Atividades Hidrolíticas e Rendimentos de Imobilização dos Biocatalisadores}

As atividades hidrolíticas e os rendimentos de imobilização obtidos de acordo com o respectivo tamanho de partícula dos suportes estudados estão dispostos na Tabela 2. As partículas retidas na peneira de 24 mesh (Tyler) e no fundo do equipamento foram descartadas para efeito de estudo.

Tabela 2 - Atividades hidrolíticas obtidas em relação aos derivados imobilizados estudados

\begin{tabular}{ccccc}
\hline & \multicolumn{4}{c}{ Suporte } \\
\cline { 2 - 5 } & \multirow{2}{c}{ STY-DVB } & \multicolumn{2}{c}{ STY-DVB magnetizado } \\
\hline \multirow{2}{*}{ Tamanho } & Atividade $\left(\mathbf{U ~ g}^{-1}\right)$ & $\begin{array}{c}\text { Rendimento } \\
(\mathbf{\eta} \%)\end{array}$ & Atividade $\left(\mathbf{U} \mathbf{g}^{-\mathbf{1}}\right)$ & $\begin{array}{c}\text { Rendimento } \\
(\boldsymbol{\eta} \%)\end{array}$ \\
\hline 35 Mesh & $1292,26 \pm 65,75$ & 64,35 & $1456,43 \pm 71,21$ & 89,39 \\
42 Mesh & $1217,42 \pm 30,44$ & 60,25 & $1295,68 \pm 58,16$ & 84,41 \\
80 Mesh & $1371,68 \pm 10,92$ & 68,47 & $1315,56 \pm 27,42$ & 80,47 \\
\hline
\end{tabular}

É possível observar pelos resultados que os dois suportes apresentaram atividades hidrolíticas semelhantes, com variação não significativa destes valores em relação ao tamanho das partículas. Estes dados permitem concluir que, entre 35 e 80 Mesh, o tamanho das partículas não influenciou no processo de imobilização.

Os rendimentos de imobilização atingiram valores significativos para ambos os suportes, indicando que os dois materiais estudados apresentam potencial para a imobilização de enzimas. Os maiores rendimentos em relação ao copolímero magnetizado se devem à menor perda de massa durante o processo de imobilização, devido à facilidade de controle das partículas através da aplicação de um campo magnético externo.

Bruno et al. (2008) atingiram 53,92\% de rendimento na imobilização de lipase de Candida rugosa em suporte magnetizado de poli(siloxano) e álcool poli(vinilico) - POS-PVA; os elevados rendimentos atingidos pelas partículas de STY-DVB magnetizado (em média $84,76 \%$ ) sugerem um grande potencial de utilização deste material na imobilização de lipases.

\section{CONCLUSÃO}

Os resultados obtidos sugerem que o suporte de STY-DVB magnetizado pode ser utilizado como suporte para imobilização de enzimas, visto que o derivado imobilizado apresentou bons rendimentos de imobilização e propriedades esperadas de magnetização. 
Foi possível concluir também que a adição de partículas magnéticas ao polímero não prejudicou sua atuação como suporte para imobilização, verificado pelas atividades hidrolíticas e seus respectivos rendimentos. Foram vistas também vantagens na separação e reutilização do biocatalisador devido à magnetização do polímero, possibilitando assim seu reuso e consequente baixa de custos operacionais.

\section{REFERÊNCIAS BIBLIOGRÁFICAS}

ADLERCREUTZ, P. Immobilisation and application of lipases in organic media. Chem. Soc. Rev. v. 42, p. 6406-6436, 2013.

BRUNO, L. M.; LIMA FILHO, J. L.; De CASTRO, H. F. Comparative Performance of Microbial Lipases Immobilized on Magnetic Polysiloxane Polyvinyl Alcohol Particles. Braz. Arch. Biol. Technol. v. 51, n. 5, p. 889-896, 2008.

COUTINHO, F. M. B.; CUNHA, L.; GOMES, L. Suportes Poliméricos para Catalisadores Sulfônicos: Síntese e Caracterização. Polímeros, v. 14, nº. 1, p. 31-37, 2004.

LEE, Y.; RHO, J.; JUNG, B. Preparation of Magnetic Ion-Exchange Resins by the Suspension Polymerization of Styrene with Magnetite. J. Appl. Polym. Sci., v. 89, p. 2058-2067, 2003.

LIU, X.; KAMINSKI, M. D.; GUAN, Y.; CHEN, H.; LIU, H.; ROSENGART, A. J. Preparation and characterization of hydrophobic superparamagnetic magnetite gel. $J$. Magn. Magn. Mater., v. 306, p. 248-253, 2006.

SOARES, C.M.F., de CASTRO, H.F., MOARES, F.F., ZANIN, G.M. Characterization and utilization of Candida rugosa lipase immobilized on controlled pore silica. Appl. Biochem. Biotechnol. , v. 77, p. 745-757, 1999. 